\title{
Binary Surfactant System for Preparation of Nano-Emulsion of Herbal Oil Mixtures
}

\author{
Usha Sayed*, Ravikant Sharma
}

${ }^{1}$ Department of Fibres \& Textile Processing Technology, Institute of Chemical Technology, Mumbai, India

\begin{abstract}
In the present study, Nanoemulsions of neem oil and pine oil using a binary surfactant system were prepared and tested their antibacterial properties. A polysorbate-type nonionic surfactant and alcohol ethoxylatesurfactant (Galaxy MW-259) were used in different proportions to obtain stable nanoemulsions at the lowest possible droplet size. The emulsification process variables such as ratio of alcohol ethoxylatesurfactant to polysorbate-type nonionic surfactant, surfactant concentration, ratio of Neem oil to pine oil, oil weight fraction and sonication time were studied. The optimal variables include the ratio of alcohol ethoxylatesurfactant to polysorbate-type non-ionic surfactant of $80: 20$, surfactant concentration of $4 \%$, ratio of Neem oil to pine oil of $80: 20$, oil weight fraction of $20 \%$ and 20 min of sonication time at $40 \%$ of the applied power. Nano-emulsions were found to be very stable with emulsion droplet size around 25-30 nm. The traditional method and probe sonicator methods are used for the preparation of oil in water Nano-emulsion of Neem oil, pine oil, a non-ionic binary surfactant system is used as an emulsifier. This newly developed nanoemulsion provides better antimicrobial, anti-bacterial and anti-mosquito repellent properties into the treated fabrics.
\end{abstract}

KEYWORDS:Nano emulsion, Binary surfactant, Alcohol ethoxylatesurfactant, Neem oil, Textile finishing

\section{INTRODUCTION}

Nano emulsions are one type of finishing chemical used in the textile industry to modify textile morphology, either physically or chemically $[1,2]$. Nano emulsions are colloidal dispersions of two immiscible phases i.e. an oil phase and an aqueous phase where emulsion droplet size ranges from 15 to $200 \mathrm{~nm}$ [3]. Nano emulsions have ignorable turbidity, good physiochemical and rheological properties [4]. Stability of Nano emulsions is very good due to very small droplet size hence creaming, flocculation, sedimentation of the particles in the emulsion does not occur for a long periods of time [5]. Droplet size of the emulsion is governed by the nature of the surfactants, type of surfactant system (single or binary), different surfactant concentration etc. [6-8].

The methodology of emulsification is also one of the vital factors to procure the smallest droplet size of the emulsion. Many researchers have focused on identifying suitable methods for emulsification which are efficient, competitive and effective $[3,7]$. Acoustic cavitation using ultrasound is one of the methods which could be employed for emulsification. Formation and subsequent collapse of micro bubbles by pressure fluctuation due to ultrasonic irradiation creates voids. The voids slowly increase in size and collapse to form a hot spot which generates localized turbulence which is responsible for the disruption of the dispersed phase into smaller droplets [9-14].

Herbal Neem (Azadirachtaindica) is recognized as a medicinal plant well known for its antibacterial and antifungal properties. At lower concentration (0.7-1 $\mathrm{mg} / \mathrm{mL}$ ) of Neem oil is found to be nontoxic while at higher concentrations $(1.2-2 \mathrm{mg} / \mathrm{mL})$ it is showing little bit toxicity. Several studies have been carried out on the emulsification of oils to utilize the unique property of the Neem oil and pine oil [15-19]. Neem is grown widely for many useful purposes, particularly as a barrier along margins of fields and in homesteads; it furnishes several worthy economic, health, and ecological benefits. The particular property that makes it interesting to use Azadirachtaindica oil is that it is rich in Azadirachtin, it's biologically active compound, which is known to influence various microbes, insects and their abilities to fly, reproduce and hormonal activities. The most significant fact is that it is found out to be non-toxic substance not only to human beings, but to other mammals as well. On the other hand, pine oil is naturally occurring herbal plant oil, generally used for the protection from mosquito bites. It is also used in the rural areas of India as herbal medicine. Pine oil can be used as an antibacterial, antifungal finishing agent $[20,21]$.

\section{MATERIALS AND METHODS 2.1 Experimental}

Pine oil and Neem oil were purchased from Peral Chemicals, Mumbai, India. Acetic acid was purchased from SD Fine Chemicals Ltd. Mumbai. Monebat -20 and Monemul-80 was purchased from Mohini Chemical ltd, Mumbai. Galaxy MW-259 ( $\mathrm{R}=\mathrm{C}_{12-14}$ ) (alcohol ethoxylate) was purchased from Galaxy Surfactant Ltd, Mumbai. Water was purified using a Millipore lab scale distilled water plant for emulsion preparation and sample preparation for analysis

\subsection{Ultrasound-Assisted Emulsification}

Nanoemulsions of Neem oil and pine oil as an oil phase wereprepared using a binary emulsifier system, acetic acid, and distilled water. Blends of alcohol ethoxylate (MW259) and polysorbate-type nonionic surfactant were used at various ratios [Alcohol Ethoxylate:polysorbate (AE:P)-0:100, 10:90, 20:80, 30:70, 40:60, 50:50, 60:40, $70: 30,80: 20,90: 10,100: 0 \%]$. Thick emulsions were prepared by mixing two different surfactants (at various concentrations: $0,0.25,0.5,1,2,3,4,5,6.5,8,10,12.5$,

\section{*Corresponding Author: ushatxt@gmail.com}


$15 \%$ of the total emulsion) with distilled water followed by the addition of mixtures of Neem and Pine oil (at different volume fractions: $0.04,0.08,0.12,0.16,0.2$ ) into the surfactant mixtures with the help of homogeniser at room temperature for 4-5 mins after complete addition of oil phase, further subjected to high energy ultrasonic emulsification. All emulsions were pre- pared at room temperature for $20 \mathrm{~min}$. The different emulsification parameters studied in the present work in order to obtain minimum particle size and stable emulsion for the longest period of time, include alcohol ethoxylate (MW259) and polysorbate ratios as mentioned above, surfactant concentrations, Neem oil and pine oil ratios [Ne:Pi$100: 0,80: 20,60: 40,40: 60,20: 80,0: 100 \%]$, volume fraction of oil in the emulsion and sonication time $(5,10$, 15, $20 \mathrm{~min})$.

\subsection{Conventional Emulsification Method}

Nanoemulsions were prepared using a high-speed homogenizer at $2500 \mathrm{rpm}$ using a 0.2 oil volume fraction and optimized surfactants' ratio and concentration at room temperature. Conventional emulsification was carried out for 40 and $50 \mathrm{~min}$.

\subsection{Mean Droplet Diameter (MDD) and Zeta Potential}

A Zetasizer Nano ZS (Malvern Instruments, UK) was used to analyze the droplet size of emulsions prepared by the above methods. This instrument is equipped with dynamic light scattering (DLS) to determine size distribution on the basis of the Brownian motion of the particles and links this to the particle size. For particle size and zeta potential samples were prepared by diluting it with the water by 1:100 ratios to obtain free Brownian motion of the samples. Equilibration time for the analysis kept to $30 \mathrm{~s}$ at $250 \mathrm{C}$. Zeta potential of the emulsion droplets was determined by evaluating the electrophoretic mobility of the particles expressed in $\mathrm{mV}$ [22].

\subsection{Emulsion Stability}

Prepared emulsions were practiced for kinetic stability using a centrifuge, and samples were selected for storage stability test which had demonstrated no phase separations or creaming upon centrifugation. Centrifugation was carried out at $2000 \mathrm{rpm}$ for $4 \mathrm{~min}$ to evaluate the kinetic stability. Stable samples were further kept for the storage stability test and periodically analysed for particles size and visually measurement of the layer of emulsion and separated material. Storage stability was conducted by storing samples at room temperature for 3 weeks. Phase separation of the Nano emulsion was determined as follows:

$$
f(\%)=\left[\frac{h_{e}}{h_{t}}\right] * 100
$$

Where the 'f (\%)' refers to the fraction of emulsion phase and ' $h e$ ' refers to the height of the emulsion and ' $h{ }^{\prime}$ ' refers to the total height of the whole emulsion system comprising the heights of the emulsion as well as the separated phase.

\section{RESULT AND DISCUSSION \\ 3.1 Surface Properties of Surfactant System}

The resistance of Neem:Pine oil Nano-emulsion droplet to deformation is managed by the interfacial tension of the surfactant system. The surface properties like surface tension, interfacial tension; contact angle etc. of the surfactant is improved by the blending of two or more surfactants. In this work, it was observed the critical micelle concentration, surface tension of the individual and mixed surfactants at various proportions in order to select surfactant judiciously for Nano-emulsion development. Also, we determined if the addition of polysorbate-type nonionic surfactant and alcohol ethoxylatesurfactant (Galaxy MW-259) altered the particle size of the emulsiondroplet stabilized by MW259 as a result of surfactant synergy.

The relationship between surfactant Galaxy MW-259: polysorbate-type nonionic surfactant CMC was determined on the basis of surface tension. Figure: 1 shows the CMC and surface tension at various surfactant blend ratios. Galaxy MW-259 shows maximum surface tension $(27.8 \mathrm{mN} / \mathrm{m})$ whereas polysorbate exhibits minimum surface tension $(21.6 \mathrm{mN} / \mathrm{m})$ because of the quick orientation at the interface and that they retain their aggregation properties at the surface results in enhancing surface tension reduction as well as CMC reduction [8].

\subsection{Surfactant System Optimization}

Emulsions were prepared using Galaxy MW-259: polysorbate-type nonionic surfactant. Alkyl alcohol ethoxylates surfactants are generally used to stabilize emulsions by tailoring the hydrophilic and hydrophobic units. Galaxy MW-259 is a nonionic surfactant having a $\mathrm{C}_{12}-\mathrm{C}_{13}$ aliphatic chain with 9 ethylene oxide units at the terminal position. Emulsions were prepared using various surfactant ratios $(0: 100-100: 0)$ of Galaxy MW-259: polysorbate-type nonionic surfactant to examine the effect of the surfactant blend ratio on emulsion droplet size at $3 \%$ surfactant concentration and $20 \%$ oil weight fraction using ultrasound-assisted emulsification.

As the contribution of Galaxy MW-259 in the blend increases, droplet size decreases slowly to a surfactant ratio of 80:20 (see Fig.2). The emulsion phase fraction (f) also increases with increasing Galaxy MW-259 up to a surfactant ratio of 80:20. Based on these results, the surfactant ratio (Galaxy MW-259: polysorbate-type nonionic surfactant) was optimized as 80:20 for further experimentation.

Emulsions were prepared using various surfactant concentrations ranging from 0.25 to $15 \%$ of the total mass of emulsion to study the effect of surfactant concentration on the emulsion stability and properties.

From Fig. 3, we can see that as the concentration of the surfactant was increased then the droplet size of Neemoil:Pine oil nano-emulsion was decreased from 280 $\mathrm{nm}$ to lowest at $26 \mathrm{~nm}$ after that more addition of surfactant from $5 \%$ to $12 \%$ it was showing negative effect on the mean droplet size.

We have studied the droplet size of an emulsion and stability using various oil proportions (Neemoil:pine oil) from 100:0 to $0: 100$ in order to examine the effect of Neemoil:Pine oil on the emulsion droplet size and ultimately on the emulsion stability. In fig: 4 we can see that the droplet size of Neem oil emulsion is less i.e. 18.3 nm compared to droplet size of Pine oil emulsion i.e. 177, $\mathrm{nm}$. So the concentration of Pine oil in oil phase is directly proportional to the droplet size of an emulsion. At Neem:Pine oil ratio of 80:20,the droplet size was seen to be $26.9 \mathrm{~nm}$, so this ratio has been considered for further experimentation. 


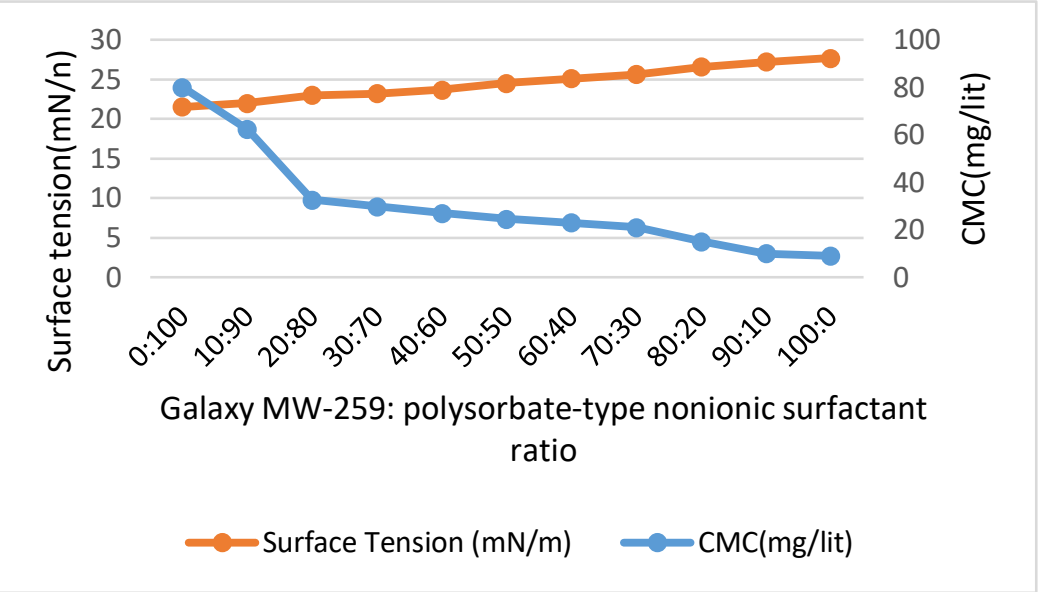

Figure 1The effect of Surfactant Ratios on Surface Tension and CMC

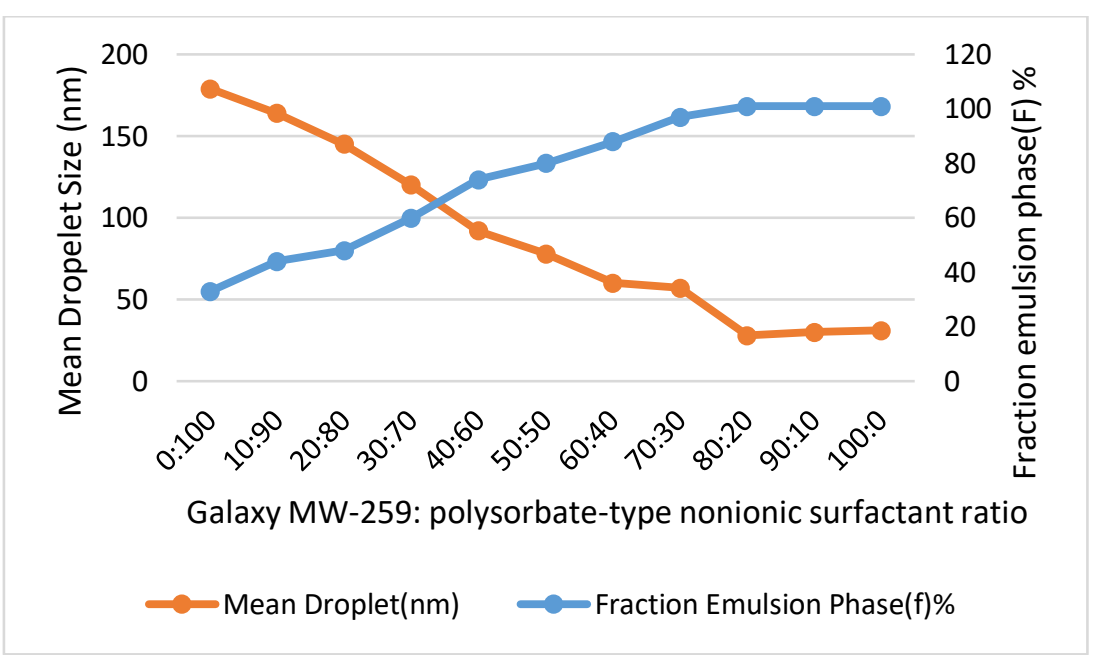

Figure 2. The effect of surfactant ratios on emulsion droplet size and the fraction emulsion phase (f) of emulsion formulated via ultrasonic emulsification method. Composition: 20\% oil weight fraction, 80:20 of Neem:Pine 0il ratio, $3 \%$ of surfactant concentration

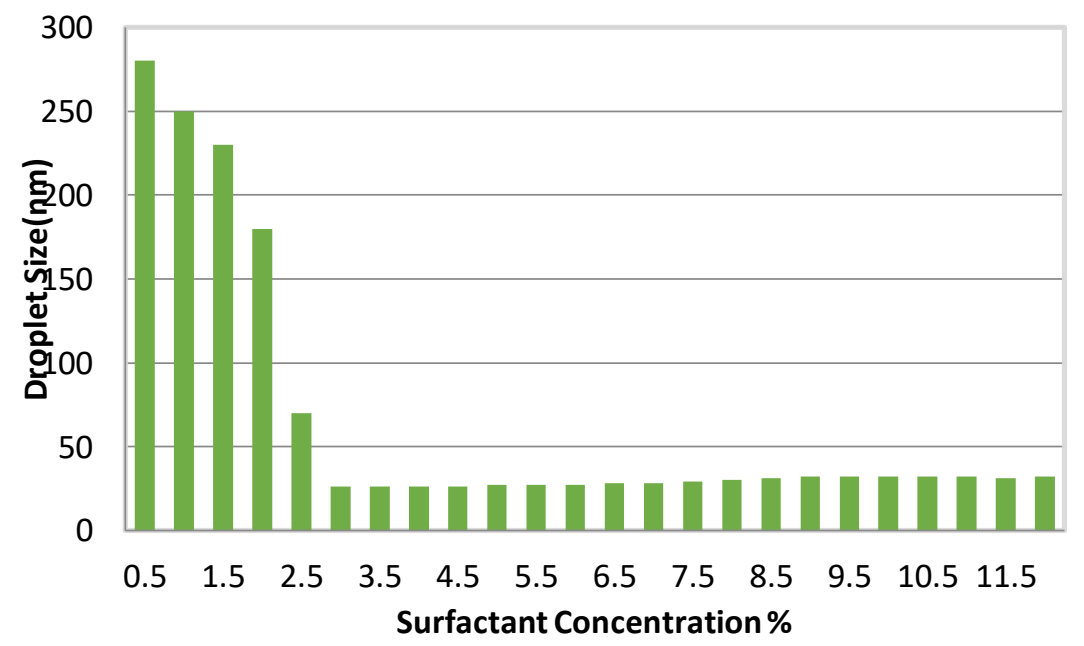

Figure 3: The effect of surfactant concentration on emulsion droplet size of emulsion formulated via ultrasonic emulsification method. Composition: 20\% oil weight fraction, 80:20 of Neem: Pine oil ratio, 80:20 of Galaxy MW259: polysorbate-type nonionic surfactant ratio 


\section{OIL RATIO AND OIL WEIGHT FRACTION OPTIMIZATION}

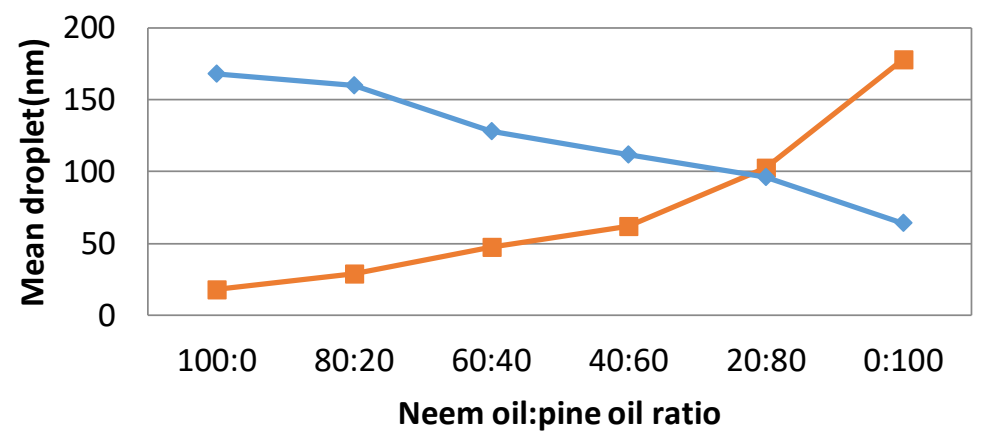

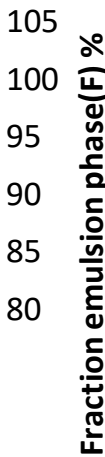

$\rightarrow$ Mean Droplet(nm) $\leadsto$ Fraction Emulsion Phase

Figure 4: The effect of Neem and pine oil ratios on emulsion droplet size and fraction emulsion phase (f) of emulsion formulated via ultrasonic emulsification method. Composition: $20 \%$ oil weight fraction, $3 \%$ of surfactant concentration, 80:20 of Galaxy MW-259 polysorbate-type nonionic surfactant ratio prepared at 40\% of applied power and 20 min sonication time

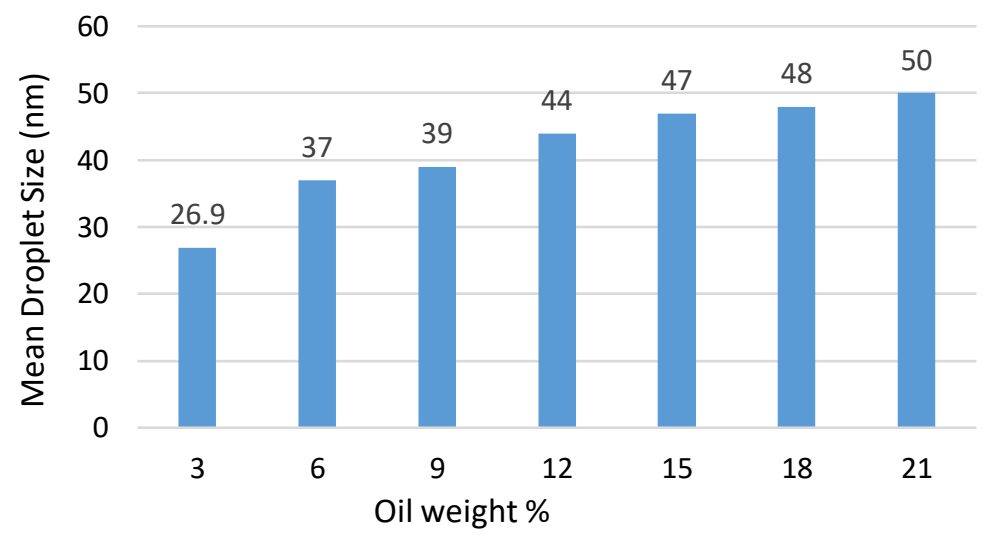

Figure 5: The effect of the \% oil weight fraction on emulsion droplet size of emulsions formulated via ultrasonic emulsification method. Composition: 3\% of surfactant concentration, 80:20 of Galaxy MW-259: polysorbate-type nonionic ratio, 80:20 of Neemoil:pine oil ratio prepared at $40 \%$ of applied power and 20 min sonication time

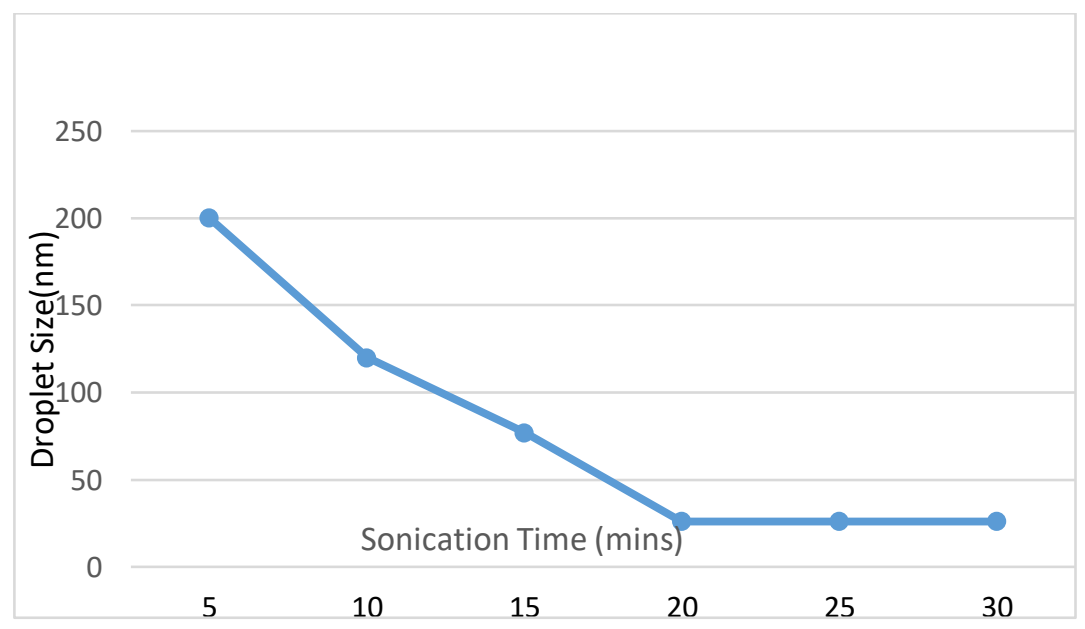

Figure 6: The effect of sonication time on emulsion droplet size of emulsion formulated via ultrasonic emulsification method. Composition: 3\% of concentration, 80:20 of Galaxy MW-259: polysorbate-type nonionic ratio surfactant ratio,80:20 of Neemoil:pine oil ratio and $20 \%$ of oil weight fraction prepared at $40 \%$ of applied power

\section{UshaSayed and Ravikant Sharma}


In Fig 5, an oil weight fraction of $3 \%$ resulted in the smallest droplet size of around $26.9 \mathrm{~nm}$. As the oil weight fraction increases from $3 \%$ to $21 \%$ then the droplet size also increases due to the higher ratio of oil to surfactant mass in the emulsion system.

From Fig 6, it can be seen that as sonication time increases from 5 mins to $20 \mathrm{mis}$ then the droplet size of emulsion decreases from $200 \mathrm{~nm}$ to $26 \mathrm{~nm}$, after that the increases in sonication time from 20 mins to 30 mins results in only an uncomparable decrease in droplet size of emulsion; hence 20 min sonication time was optimized from the experiments.

\section{RESULTS AND DISCUSSION}

For the purpose of preparing the nanoemulsion, two methods were selected namely, High Speed homogenisor assisted method, which is the traditional method and is a completely physical mixing and the final product formed solely depends on the RPM at which the setup is run. And the other method, more modern method is the Ultrasound assisted emulsification, acoustic cavitation has been employed to achieve the smallest possible droplet size at the shortest time span. The point to remember here is that Neem oil and Pine oil have different densities, and also are not miscible. Also notable is the fact is that the oil and aqueous phase are also not miscible. In order to make a homogeneous system from these three phases, an energy intensive technique is required to obtain the desired droplet size. When sonication is employed, the rate of energy dissipation in the system increases which results in increased temperature. Ultrasonication is also responsible for the production of shear forces which causes breakdown of large droplets into smaller droplets and newly generated droplets are stabilized by surfactant.

The purpose of using a binary system is to incorporate the properties of both the oils into the emulsion system. Thus it is of prime importance that the system so produced is very stable. And that's why it was very important to choose the right kind of surfactant mixture to stabilize this highly hydrophobic mixture. Based on the previous works it was identified that Galaxy MW-259: polysorbate-type nonionic surfactant are better suited for a mixture containing neem oil and then depending on the HLB (Hydrophile-Lipophile Balance) calculations it was found out that the Lauryl alcohol ethoxylates, were the ones meeting the requirements. Thus it was concluded to carry out the emulsification process with a Binary surfactant system, containing both non-ionic surfactants, because that gave the best compatibility.

Final product was made as Oil-in-Water emulsion as Oil was dispersed phase and had to be emulsified. So how to meet this 'Required HLB' value is by choosing a proper surfactant which has its own HLB value in the similar range. Most of the Oil-in-Water systems require surfactants with HLB in range of 8-16. But here a mixture of surfactants is being used because it has been found out that mixture is better than using single surfactant, as it helps us adjust the price and viscosity of the product as well.

\section{CONCLUSION}

The preparation of oil in water Nano emulsions of Neem oil and pine oil i.e, herbal oil mixtures has been successful studied for thefollowing reasons:

1. Due to the use of Sonicator Probe
2. Due to the use of Binary surfactant systems

3. A polysorbate non-ionic surfactant and alcohol Ethoxylate surfactant was studied for the first time for nano-emulsion preparation of Neem oil and Pine oil mixture.

4. The ultrasound-assisted emulsion of Neem oil and pine oil stabilized by a binary system of surfactant was prepared successfully withlowest possible droplet i.e. $26.9 \mathrm{~nm}$.

5. Although nano emulsion can be produced by any level of parameters optimization all the required variables for the developmentof stable emulsion within desired particle size was successfully studied.

6. The optimal variables include the ratio of alcohol ethoxylate surfactant to polysorbate-type non-ionic surfactant of $80: 20$, surfactant concentration of $3 \%$, ratio of Neem oil to pine oil of 80:20, oil weight fraction of $20 \%$ and $20 \mathrm{~min}$ of sonication time at $40 \%$ of the applied power. Nano-emulsions were found to be very stable with emulsion droplet size around $25-30 \mathrm{~nm}$.

\section{REFERENCES}

[1]. Radhakrishnan S. Roadmap to sustainable textiles and clothing.2014. Springer, Singapore.

[2]. Chandrasekar S, Vijayakumar S, Rajendran R, Rajesh R, Elayarajah B, 2013.Herbal-chitosan nanocomposites for durable antibacterial finishing on cotton materials, International Journal of Biopharmaceuticals,4(3), 219-224

[3]. Abbas S, Bashari M, Akhtar W, Li WW, Zhang X. 2014. Processoptimization of ultrasound-assisted curcuminnanoemulsions stabilized by OSA-modified starch. UltrasonSonochem21:1265-74.

[4]. Barry BW, Polytechnic P, Street KHI, Kingdom U.1976.Surfaceproperties and micelle formation of long-chain polyoxyethylenenonionic surfactants. J Colloid Interface Sci. 54:339-47.

[5]. Tadros TF. Emulsion formation, stability, and rheology. EmulsForm Stab. 2013. doi:10.1002/9783527647941.ch1.

[6]. Bardhan S, Kundu K, Saha SK, Paul BK.2013.Physicochemicalstudies of mixed surfactant microemulsions with isopropylmyristate as oil. J Colloid Interface Sci. 402:180-9..

[7]. Lin L-H, Wang C-C, Chen K-M, Lin P-C. 2013. Synthesis andphysicochemical properties of siliconbased geminisurfactants.Colloids Surf A PhysicochemEng Asp. 436:881-9.

[8]. Sastry NV, Punjabi SH, Ravalji IR.2013. Effect of toctylphe-noxylpolyethoxyethanol (TX-100) on the dilute aqueous solutionphase diagrams, surface activity and micellizationbehaviorofnon-ionic silicone surfactants (SS) in aqueous media. J Mol Liq.177:215-24.

[9]. Liu M, Ragheb AN, Zelisko PM, Brook MA. 2014. Preparation andapplications of silicone emulsions using biopolymers. SurfactantSciSer, 116:309-27.

[10]. Ghosh V, Mukherjee A, Chandrasekaran N,2013. Ultrasonic emulsifi-cation of food-grade nanoemulsion formulation and evaluation ofits bactericidal activity. UltrasonSonochem. 20:338-44. 
[11]. Singh BP, Pandey BP, 1992.Ultrasonication for breaking water-in-oilemulsion.Proc Indian NatlSci Acad. 58:181-94.

[12]. Jadhav AJ, Holkar CR, Karekar SE, Pinjari DV, Pandit AB. 2015.Ultrasound assisted manufacturing of paraffin wax nanoemulsions: process optimization. UltrasonSonochem. 23:201-7.

[13]. Challis RE, Povey MJW, Mather ML, Holmes AK.2005. Ultrasoundtechniques for characterizing colloidal dispersions. Rep Prog Phys. 68:1541-637.

[14]. Pukale DD, Maddikeri GL, Gogate PR, Pandit AB, Pratap AP,2015.Ultrasound assisted transesterification of waste cooking oil usingheterogeneous solid catalyst. UltrasonSonochem. 22:278-86.

[15]. JayakumarJerobin, PoojaMakwana, RS Suresh Kumar, Rajiv Sundaramoorthy, Amitava Mukherjee, and Natarajan Chandrasekaran,2015. Antibacterial activity of neemnanoemulsion and its toxicity assessment on human lymphocytes in vitro, Int J Nanomedicine. 10(Suppl 1): 77-86.

[16]. Chandrasekar S, Vijayakumar S, Rajendran R, Rajesh R, Elayarajah B,2013. Herbal chitosan nanocomposites for durable antibacterial finishing on cotton materials, International Journal of Biopharmaceutics. 4(3): 219-224.

[17]. Sugumar S, Singh S. 2016.Nanoemulsion of orange oil with non-ionicsurfactant produced emulsion using ultrasonication techniqueevaluating against food spoilage yeast. ApplNanosci. 6(1) 113-120

[18]. Kothekar SC, Momin SA. 2008. Formulating neem oil emulsion aspotent agrochemicals using a binary emulsifier system. J DispersSci Technol. 29:919-29.

[19]. Taylor E, Webster TJ. 2011. Reducing infections through nanotechnology and nanoparticles. IntJNanomedicine. 6:1463-1473.

[20]. Ansari MA, Mittal PK, Razdan RK, Sreehari U.2005.Larvicidal and mosquito repellent activities of Pine (Pinuslongifolia, Family:Pinaceae) oil. J. Vector Borne Dis. 42:95-9.

[21]. Ferreira Maia M, Moore SJ, 2011. Plant-based insect repellents: areview of their efficacy, development and testing. Malar J. 10:S11.

[22]. Dipak D. Pukale,Archana S. Bansode, Dipak V. Pinjari1,Usha Sayed, Rahul R. Kulkarni, 2017. Development of Nanoemulsion of Silicone Oil and Pine Oil UsingBinary Surfactant System for Textile Finishing, Journal of Surfactants and Detergents 20 (5), 1061-1073 